\title{
Estilos e Estratégias de Aprendizagem Personalizadas a Alunos das Modalidades Presenciais e a Distância
}

\author{
Title: Styles and Learning Strategies Personalized for Students of Traditional and Distance education \\ Andreia Rosangela Kessler Mühlbeier \\ UNICRUZ - Universidade de Cruz Alta \\ Cruz Alta - RS \\ andreiamuhlbeier@yahoo.com.br \\ Patricia Mariotto Mozzaquatro \\ UNICRUZ - Universidade de Cruz Alta \\ Cruz Alta - RS \\ patriciamozzaquatro@gmail.com
}

Resumo A aprendizagem vem a cada dia tomando impulso gradativo nas formas de educação, a utiliza-
ção das tecnologias de informação e comunicação (TICs) e de um modo particular a internet,
constitui um dos eixos orientadores desse desenvolvimento. Neste contexto, mais importante do
que as TICs é a forma com que as mesmas são utilizadas no cotidiano das pessoas, ou seja,
conforme suas características individuais. Este artigo apresenta o desenvolvimento de um Sis-
tema para diagnosticar estilos de aprendizagem, podendo assim, possibilitar e promover situa-
ções de aprendizagem individualizadas e direcionadas, ou seja, o professor poderá ter informa-
ções sobre o seu próprio estilo de aprendizagem e conhecer as preferências de aprendizagem de
seus alunos a fim de desenvolver as complementaridades e obter elementos que sirvam para
diferenciar a sua prática pedagógica. O sistema foi validado com discentes dos cursos de gra-
duação presenciais em Ciências Contábeis, Medicina Veterinária, Pedagogia e docentes do
Curso de Capacitação no Ambiente Virtual de Aprendizagem Moodle (modalidade a distância)
da Universidade de Cruz Alta. Após, foi diagnosticado através de uma amostra os quatro estilos
de aprendizagem de maior predominância. A partir da análise desses resultados foi gerado um
estudo de caso, definindo metodologias e técnicas de apresentação de materiais.

Palavras-Chave: estilos de aprendizagem, estratégias de aprendizagem, materiais educacionais.

\begin{abstract}
The learning is gaining momentum every day in gradual forms of education, the use of information technologies and communication technologies (ICTS) and in a particular way the Internet is one of the guiding axes of development. In this context, most important of which ICT is the way they are used in daily life, ie according to their individual characteristics. This article presents the development of a system to diagnose learning styles and may thus enabling and promoting individualized learning situations and directed, that is, the teacher might have information about your own learning style and meet the learning preferences of their students to develop complementarities and get items that serve to differentiate their practice. The system was validated with the undergraduate students face in Accounting Sciences, Veterinary Medicine Teaching and Teachers Training Course in Moodle Virtual Learning Environment (distance mode) at the University of Cruz. Alta. After he was diagnosed through a test the four learning styles most predominant. From the analysis of these results was generated a case study, defining methodologies and techniques of presentation of materials.
\end{abstract}

Keywords: learning styles, learning strategies, educational materials. 


\section{Introdução}

Nas últimas décadas, segundo [1] "a educação à distância (EAD) tomou um novo impulso que favoreceu a disseminação e a democratização do acesso à educação em diferentes níveis e formas de interação e aprendizagens".

Nesse sentido, a utilização das tecnologias de informação e comunicação (TICs) e de um modo particular a internet, constitui um dos eixos orientadores do desenvolvimento da educação e formação, podendo ser considerada como um dos paradigmas de inovação e evolução das sociedades. No entanto, mais importante do que qualquer tecnologia é a forma como as pessoas a utilizam no seu desenvolvimento individual ou coletivo, ou seja, como as pessoas tornam as tecnologias úteis às suas vidas, tendo em conta os seus estilos de aprendizagem [9].

Felder afirma que:

Estilos de aprendizagem podem ser definidos como as características internas ou as preferências individuais dos aprendizes na forma de receber e/ou processar informações. Tais estilos, nem sempre conscientes, também exercem influência marcante nas estratégias utilizadas para aprender [4].

Quando o docente tem conhecimento sobre o estilo de aprendizagem do discente facilita o desenvolvimento e a utilização de metodologias e técnicas de ensino, motivando assim a geração de melhores resultados, na aprendizagem do aluno.

O presente artigo busca desenvolver um estudo aprofundado sobre as dimensões dos estilos de aprendizagem que compõe o modelo do aluno. Foi desenvolvido um sistema para diagnosticar estilos de aprendizagem. O mesmo embasou-se nos modelos propostos por [2, 4, 5, 6], possibilitando assim, situações de aprendizagem individualizadas e direcionadas.

\section{Estilos de Aprendizagem}

Sabe-se que identificar os estilos de aprendizagem dos alunos é importante, uma vez que pode explicar o porquê de certas estratégias de aprendizagem funcionarem bem e outras não.

De acordo com [3]:

Os estilos de aprendizagem são a maneira com que o aprendiz utiliza estratégias de aprendizagem na construção do conhecimento. Tais estratégias são ferramentas que o sujeito desenvolve para lidar com diferentes situações de aprendizagem incompatíveis com seu estilo [3].
Portanto, quanto mais estratégias o aluno tiver desenvolvido, maior será a chance de lidar com as diversas formas de apresentação das informações nas situações de aprendizagem por ele vivenciadas.

Conforme [7]:

\begin{abstract}
A identificação dos estilos de aprendizagem é importante no sentido de incitar uma ligação entre o ensino e os modos como os alunos preferem aprender e, se assim for, os alunos demonstram melhores resultados e um desejo mais forte de aprender [7]
\end{abstract}

Desse modo, o professor, apesar de planificar atividades que desafiem os alunos a desenvolver as suas capacidades, devem ter em atenção os seus estilos de aprendizagem.

\subsection{As dimensões dos estilos de aprendizagem}

Quanto às dimensões dos estilos de aprendizagem, um grande número delas encontra-se disponível na literatura. Podem ser citadas as seguintes, de maior relevância.

\subsubsection{Modelo Butler}

O modelo de Butler apresenta cinco dimensões de estilos de aprendizagem: Realista, Analítico, Pragmático, Pessoal e Divergente (Figura 1).

De acordo com [2]: Estilo de aprendizagem é a forma consistente e pessoal através da qual as pessoas usam suas qualidades e habilidades para definir a si mesmas, para encontrar, avaliar e processar informações.

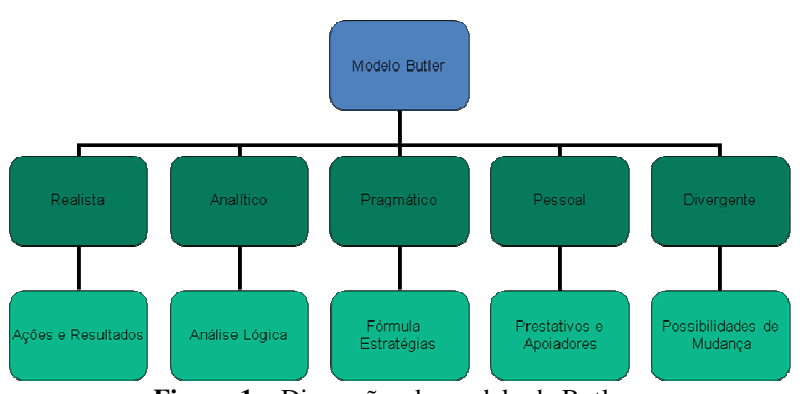

Figura 1 - Dimensões do modelo de Butler. 


\subsubsection{Modelo Honey e Munford}

O modelo proposto por Honey e Munford considera que as principais características das pessoas podem ser integradas em quatro estilos de aprendizagem: Ativo, Reflexivo, Teórico e Pagmático (Figura 2). Admitem ainda, que "em cada pessoa é possível identificar características dos vários estilos de aprendizagem, embora geralmente, cada pessoa possua um estilo dominante" [5]. Foi criado o Cuestionario Honey-Alonso de Estilos de Aprendizaje (CHAEA), na versão Portuguesa, com o objetivo de ajudar os alunos a estarem atentos aos seus pontos fortes e fracos dos estilos de aprendizagem.

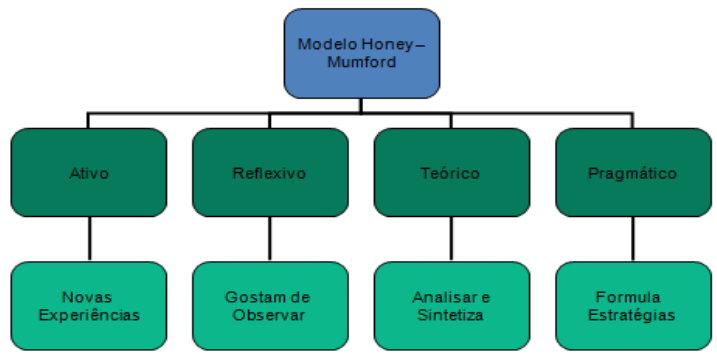

Figura 2 - Dimensões do modelo de Honey e Munford.

\subsubsection{Modelo Felder e Silvermann}

Richard M. Felder e Barbosa A. Soloman baseados em diversas pesquisas de outros autores, formularam um modelo de estilos de aprendizagem com dimensões relevantes para a educação científica. Os autores definem "estilos de aprendizagem como preferências e características dominantes no modo como as pessoas recebem e processam as informações" [4]. O modelo proposto define quatro dimensões de estilos: Retenção da informação (Visual-Verbal), Percepção da informação (SensorialIntuitivo), Processamento da informação (AtivoReflexivo) e Organização da informação (SeqüencialGlobal) (Figura 3). Para a identificação dos estilos de aprendizagem propostos no modelo Felder-Silverman foi desenvolvido um instrumento, denominado Index of Learning Styles Questionnaire (ILS).

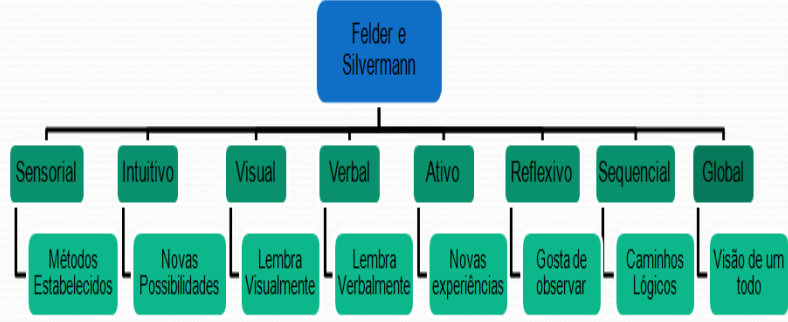

Figura 3 - Dimensões do modelo de Felder e Silvermann.

\subsubsection{Modelo Kolb}

Segundo [6] observa que cada sujeito desenvolve um estilo de aprendizagem particular, dando prioridade a certa habilidade. A partir da combinação das quatro habilidades descritas, ele propõe uma categoria dos estilos de aprendizagem: divergente, assimilador, convergente, acomodador (Figura 4).

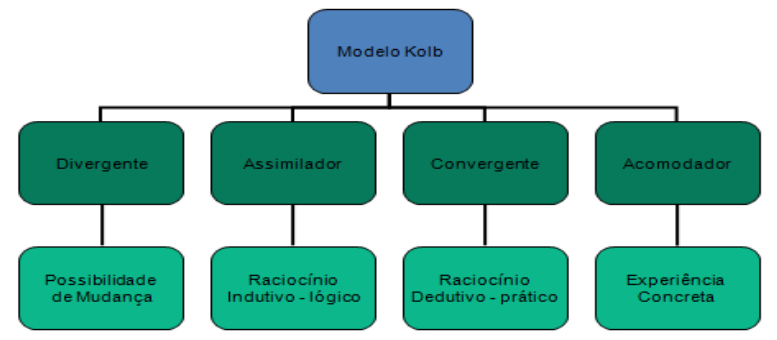

Figura 4 - Dimensões do modelo de Kolb.

O estilo de aprendizagem refere-se às preferências do sujeito no processo de aprendizagem. Partindo das pesquisas realizadas sobre estilos de aprendizagem e, que o termo estilo pode dar a ideia de uma marca definitiva, [3] acredita:

\footnotetext{
Ser mais adequado utilizar o termo preferências de aprendizagem para denominar o conjunto de preferências que determina uma abordagem individual para aprender, nem sempre compatível com as situações de aprendizagem. Estas preferências variam ao longo da vida, de acordo com a situação de aprendizagem, seu conteúdo e a experiência do apren$\operatorname{diz}[3]$.
}

Assim, identificando a preferência de aprendizagem do aluno é possível elaborar estratégias de aprendizagem mais eficazes.

Diante do exposto, a autora [8] cita em sua tese que o ideal seria haver um equilíbrio, propondo ao aluno 
atividades que vão ao encontro de suas preferências individuais e que lhe desafiem a experimentar certo desconforto, fazendo com que desenvolva novas estratégias de aprendizagem.

\section{Descrição do experimento}

O experimento consistiu no desenvolvimento e aplicação de um sistema para diagnosticar estilos de aprendizagem de alunos das modalidades presenciais e a distancia. A modelagem proposta foi realizada através de diagramas UML, uma linguagem destinada a visualizar, especificar, construir e documentar sistemas de software. Os diagramas utilizados são os diagramas de casos de uso e atividades.

Num primeiro momento, o aluno deverá acessar o SDLE - System Detector Learning Styles, disponível em: $<$ http://ideal.inf.br/moodle/andreia/index1.php>. Após será apresentado um questionário o qual deve ser preenchido. Esse questionário tem como função identificar o estilo de aprendizagem predominante do aluno, relacionado as dimensões já citadas na seção anterior. A Figura 5 apresenta o Caso de Uso geral na visão dos usuários. Num primeiro momento o aluno irá acessar o sistema respondendo o questionário, que compõem-se de 17 dimensões de estilos de aprendizagem. Após concluída está etapa será apresentado o resultado com o estilo detectado onde aluno e professor poderão ter conhecimento.

O diagrama de atividades apresentado na Figura 6, subdividi-se em 3 (três) papeis: docente, sistema e discente. O discente acessa o sistema, responde o questionário, o sistema identifica o estilo de aprendizagem apresentado resultado ao discente. Também o docente poderá ter acesso ao sistema visualizando o resultado detectado. O estilo de aprendizagem do aluno será identificado através do instrumento "Questionário" embasado nos instrumentos de investigação propostos por [2, 4, 6].

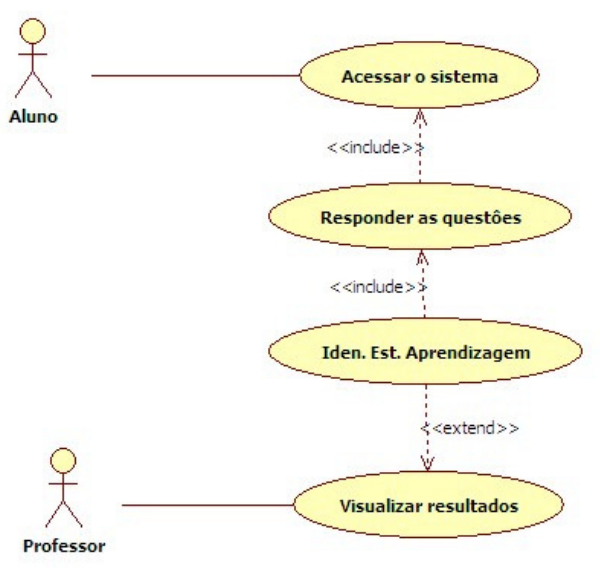

Figura 5 - Diagrama de Caso de Uso.

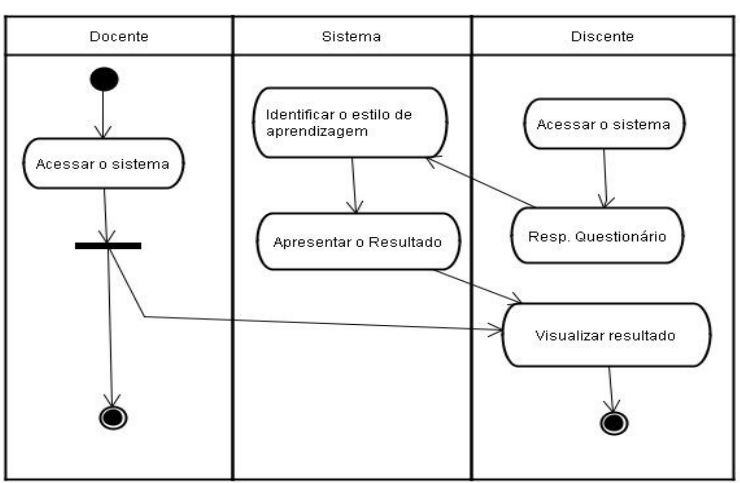

Figura 6 - Diagrama de Atividades.

O resultado do teste indicará as preferências em relação as seguintes dimensões: Acomodador, Analítico, Assimilador, Ativo, Convergente, Divergente, Global, Intuitivo, Pessoal, Pragmático, Realista, Reflexivo, Sensorial, Sequencial, Teórico, Verbal, Visual. O sistema foi constituído por cinqüenta questões objetivas (três questões de cada dimensão). Nas questões objetivas o aluno deve escolher uma das quatro alternativas apresentadas (Concordo), (Concordo Totalmente), (Discordo) e (Discordo Totalmente) aquela que mais se aproxima da sua atitude ou do seu comportamento na situação apresentada.

O sistema proposto apresentou as seguintes funcionalidades: armazenar as informações após o preenchimento do questionário; determinar a média da pontuação obtida a cada estilo; comparar as médias obtidas, apresentando o Estilo correspondente a maior média; identificar e, registrar o nível de preferência em cada um dos estilos referidos. O sistema intitulado SDLE na qual são especificadas informações relacionadas ao significado de Estilos de Aprendizagem e orientações sobre o preenchimento do questionário e validação do sistema. 

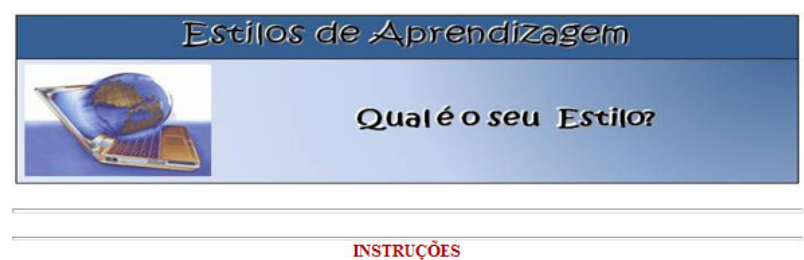

Pense no que acontece com maior frequência. Por faror, para cada questão marque uma das alternativas e não deixe nenhum item sem resposta.

Legendas utilizadas:

$\begin{array}{ccc}\text { DT - } & \text { D- } & \text { C- } \\ \text { DISCORDO } & \text { CT- } \\ \text { DISCORDO CONCORDO CONCORDO }\end{array}$

pós responder a todas as questões, clique no Botão Confirmar.

1. Se eu fosse professor, eu preferiria ensinar uma disciplina que trate com fatos e situaçoes reais.

Discordo Totalmente Discordo Concordo Concordo Totalmente

Figura 7 - Tela do Questionário.

Após obter informações sobre o preenchimento do questionário, o participante irá responder as 51 questões objetivas com quatro alternativas de resposta: Discordo Totalmente (DT), Discordo(D), Concordo Totalmente (CT) e Concordo(C), conforme a Figura 7.

Após finalizar o preenchimento do questionário, o participante deverá clicar no botão Confirmar, visualizando assim, seu estilo de aprendizagem, como também, o conceito do respectivo Estilo de Aprendizagem detectado Figura 8.

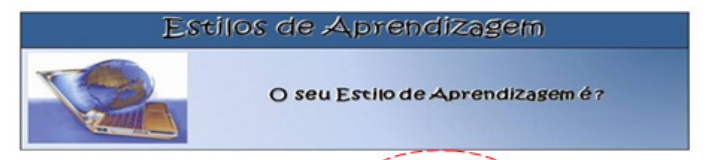

SEU ESTHL DE APRENDIZAGEMIÉ ESTHO ATIVO

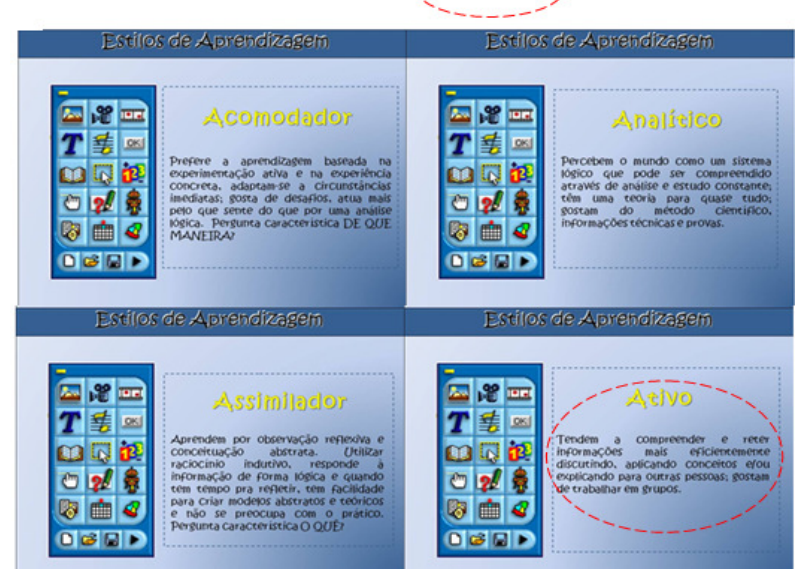

Figura 8 - Resultado do Estilo detectado.
Para diagnosticar o estilo de aprendizagem foi realizado um critério de distribuição entre as perguntas no questionário. Foram distribuídas três questões correspondente a cada dimensão integrante dos modelos propostos pelos autores pesquisados, conforme a Tabela 1 .

Tabela 1 - Distribuição das Questões por dimensão

\begin{tabular}{l|l}
\hline \multicolumn{1}{c|}{ Questöes } & \multicolumn{1}{c}{ Dimensåo dos Estilos de Aprendizagem } \\
\hline $1,2,3$ & Estilo Sensorial \\
\hline $4,5,6$ & Estilo Intuitivo \\
\hline $7,8,9$ & Estilo Visual \\
\hline $10,11,12$ & Estilo Verbal \\
\hline $13,14,15$ & Estilo Sequencial \\
\hline $16,17,18$ & Estilo Global \\
\hline $19,20,21$ & Estilo Divergente \\
\hline $22,23,24$ & Estilo Assimilador \\
\hline $25,26,27$ & Estilo Convergente \\
\hline $28,29,30$, & Estilo Acomodador \\
\hline $31,32,33$ & Estilo Ativo \\
\hline $34,35,36$ & Estilo Reflexivo \\
\hline $37,38,39$ & Estilo Térico \\
\hline $40,41,42$ & Estilo Pragmático \\
\hline $43,44,45$ & Estilo Realista \\
\hline $46,47,48$ & Estilo Analitico \\
\hline $49,50,51$ & Estilo Pessoal \\
\hline
\end{tabular}

Para detectar seu estilo de aprendizagem, cada usuário deverá responder o questionário optando por quatro alternativas traduzidas por Discordo Totalmente, Discordo, Concordo e Concordo Totalmente. Foi determinado que a pontuação de cada item corresponde a respectivamente 1 , 2, 3 ou 4. A pontuação resultante do estilo de aprendizagem do usuário em cada estilo é obtida pelo somatório dos números que revelam as suas respostas na totalidade dos itens relacionados a cada estilo, conforme a Figura 9.

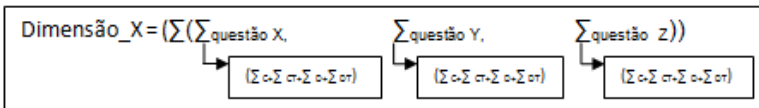

Figura 9 - Pontuação Estilo de Aprendizagem.

O critério utilizado na pontuação dos estilos de aprendizagem foi embasado no instrumento proposto por [5]. Para visualizar o nível de preferência de cada aluno nos respectivos estilos de aprendizagem foi realizado um somatório das pontuações obtidas, totalizando uma média. Após foi realizado um comparativo entre as médias correspondentes aos dezessete estilos de aprendizagem. A maior média originou o estilo predominante. Conforme apresentado na Tabela 1 cada dimensão dos estilos de 
aprendizagem foi composta de 3 questões distribuídas aleatoriamente, totalizando 51 questões, dentre os 17 estilos de aprendizagem. Para detectar o estilo de maior predominância foi realizado um somatório correspondente a cada dimensão, para após ser comparada as médias apresentando a dimensão predominante.

\section{Resultados e Discussão}

Com base nos dados coletados foram gerados gráficos para apresentar os resultados da pesquisa. O SDLS (System Detector Learning Styles) foi aplicado a setenta discentes e docentes integrantes das modalidades presenciais e a distância. Conforme a Figura 10 participaram da pesquisa discentes dos Curso de graduação presenciais Ciências Contábeis (16\%), Medicina Veterinária (14\%), Pedagogia (13\%) e o curso à distância de Capacitação Moodle (57\%).

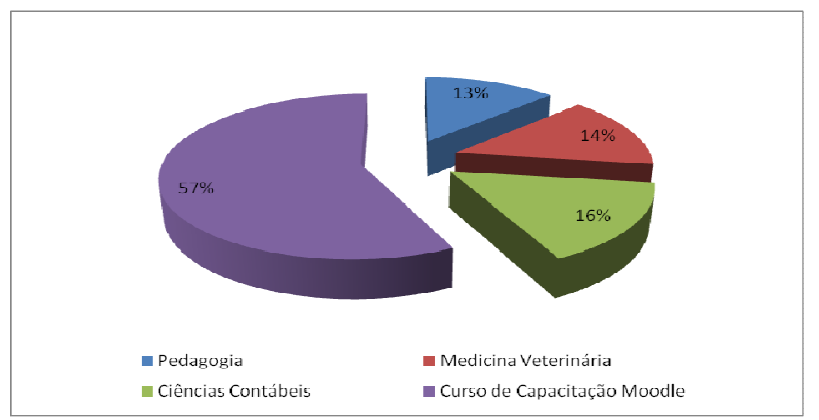

Figura 10 - Cursos Entrevistados.

Em relação aos estilos de aprendizagem de maior predominância destacaram-se o Estilo Visual, Global, Verbal e Sequencial. Em uma menor proporção, os estilos Acomodador, Analítico, Assimilador, Reflexivo, Ativo, Convergente, Divergente, Intuitivo, Pessoal, Pragmático, Realista, Sensorial e Teórico. Conforme demonstra o Figura 11.

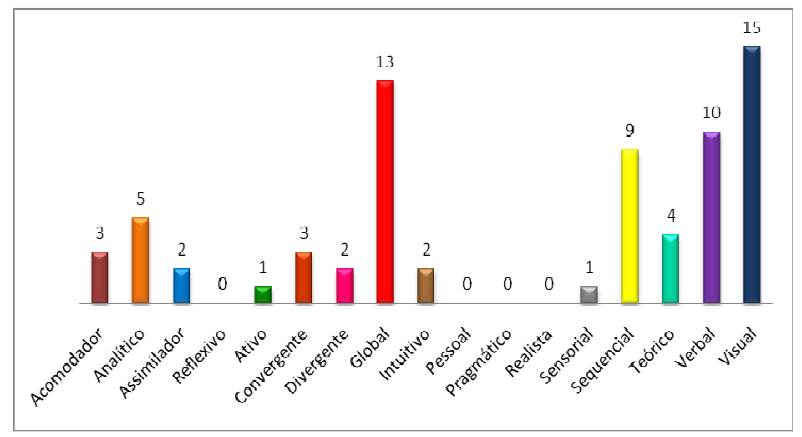

Figura 11 - Estilos de Aprendizagem estudados.
Conforme demonstra a Figura 12 os quatro estilos de aprendizagem de maior predominância, destacaram-se como Estilo Visual (32\%), seguido do Estilo Global (28\%), Estilo Verbal (21\%) e Estilo Sequencial (19\%).

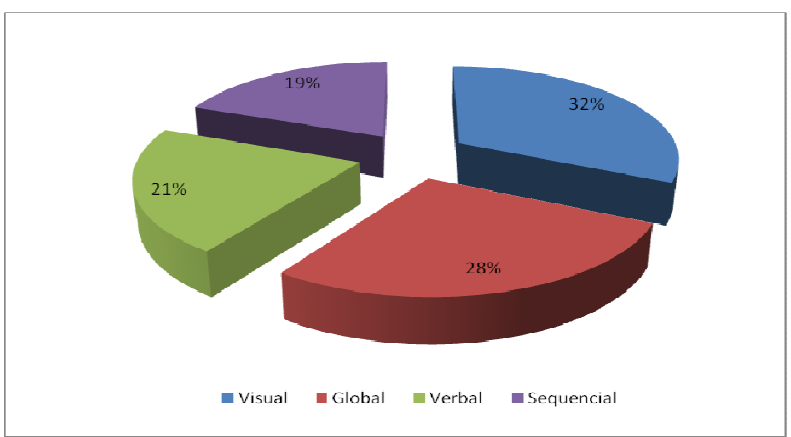

Figura 12 - Estilos de Aprendizagem de maior predominância.

\subsection{Indicadores para o desenvolvimento de Métodos e Estratégias de Aprendizagem}

No resultado apresentado no sistema para diagnosticar estilos de aprendizagem, definiu-se métodos e estratégias relacionados a forma de apresentação de conteúdos, atividades e ferramentas direcionadas a cada estilo detectado. A seguir Figura 13, são apresentadas características dos quatro estilos de maior predominância, segundo os autores [4].

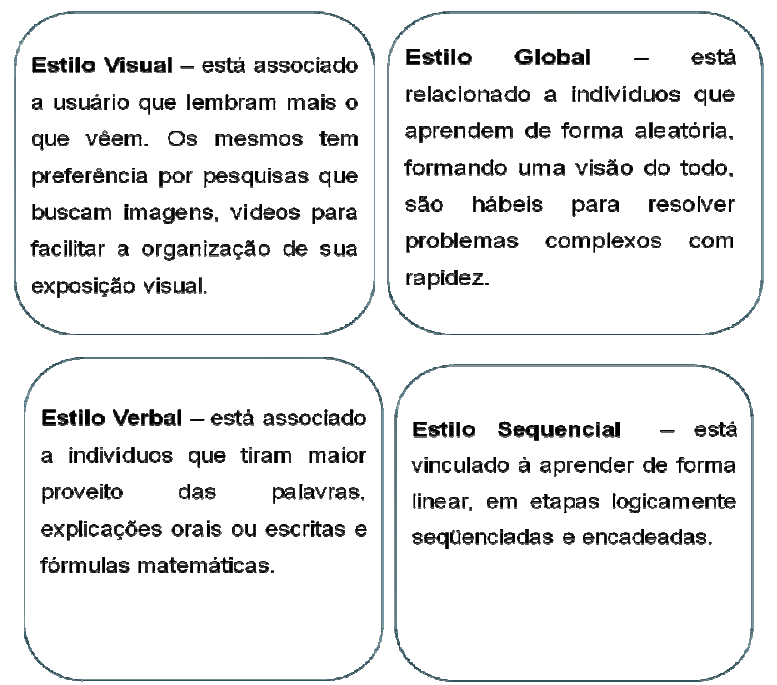

Figura 13 - Características dos Estilos de Aprendizagem de maior predominância. 


\subsection{Materiais Instrucionais para o Desenvol- vimento de Estratégias de Aprendizagem}

Foram estabelecidas 27 técnicas e métodos, sendo 11 técnicas de conteúdo, 06 atividades, 05 ferramentas e 05 estratégias pedagógicas, e definidas quais as mais adequadas para cada estilo de aprendizagem.

A Figura 14 apresenta material adaptado ao Estilo de Aprendizagem Visual, demonstrando a classificação, conceito e característica de um software educativo na forma de diagrama.

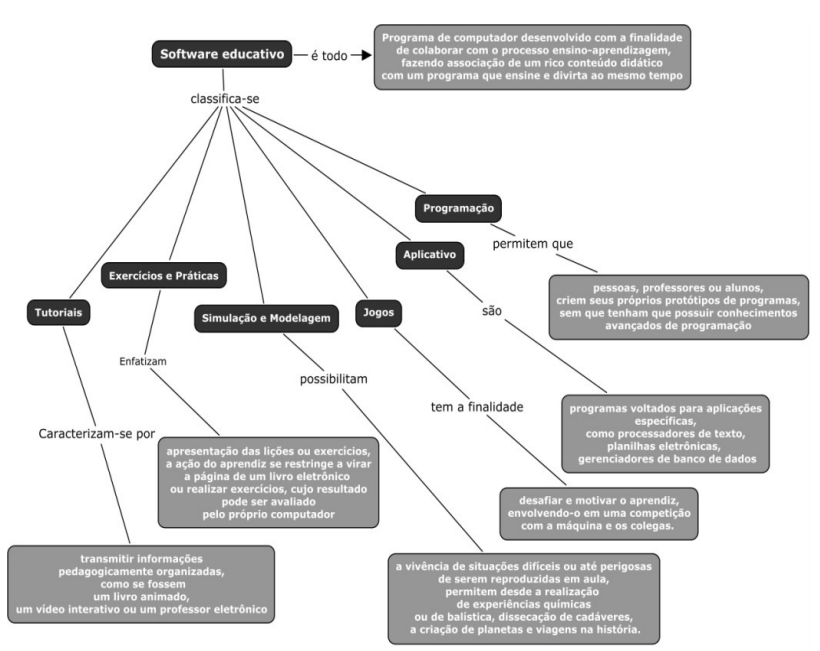

Figura 14 - Material instrucional estilo de aprendizagem Visual.

\section{Facilidade de uso}

Relativo à objetividade das instruçöes para o uso do SE e à facilidade de percorrê-lo.

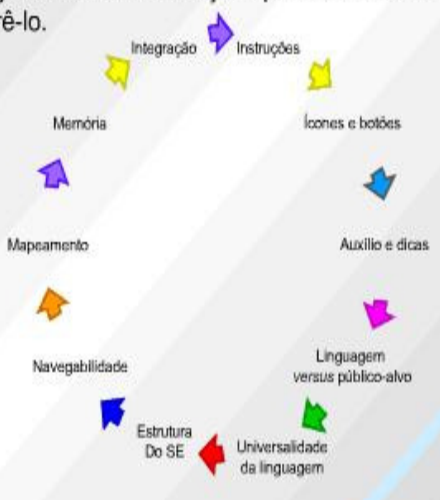

Figura 15 - Material instrucional estilo de aprendizagem Global.

Na Figura 15, ainda tratando-se do tema software educativo e apresentado um esquema, formato de material preferencial integrante do Estilo de Aprendizagem Global.

A Figura 16 ilustra um software educativo em forma de vídeo o qual a apresenta-se como material preferencial ao Estilo de Aprendizagem Verbal.

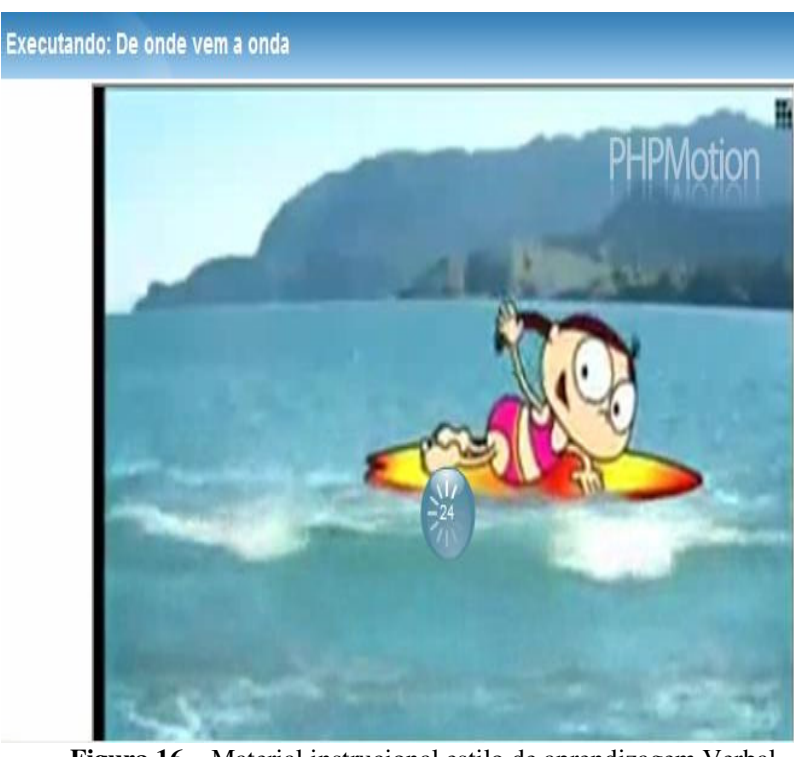

Figura 16 - Material instrucional estilo de aprendizagem Verbal.

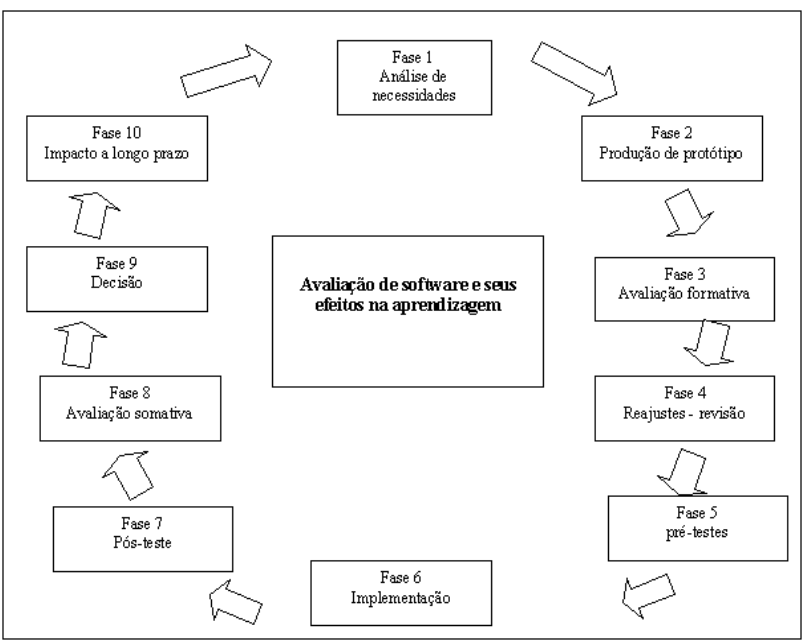

Figura 17 - Material instrucional estilo de aprendizagem Sequencial.

E na Figura 17, ainda abordando softwares educativos é apresentado um esquema ilustrando as fases de avalia- 
ção de software e seus efeitos na aprendizagem, material preferencial ao estilo de Aprendizagem Sequencial.

\section{Considerações Finais}

Para o desenvolvimento do trabalho proposto formam realizados estudos sobre os modelos e dimensões existentes sobre Estilos de Aprendizagem embasados nos seguintes autores $[2,4,5,6]$. Após foi desenvolvido um sistema computacional que integrou dezessete dimensões de estilos de aprendizagem retornando ao usuário seu estilo preferencial.

Com os dados validados através do SDLE, definiu-se os quatro estilos de aprendizagem de maior predominância (visual, verbal, global e sequencial), para assim ser realizado um estudo aprofundado sobre estratégias e métodos de apresentação de conteúdo conforme os estilos detectados. Este trabalho corrobora com outras pesquisas as quais também concluíram que diagnosticar estilos de aprendizagem é uma tarefa complexa, e requer estudos aprofundados para que possa ser oferecida ao aluno uma experiência de aprendizagem individualizada, apresentando-lhe a informação de maneira personalizada e direcionada. A partir dos dados coletados foi possível identificar os estilos preferenciais dos alunos, possibilitando assim, efetivar indicadores para possível organização do conteúdo orientando na construção de materiais instrucionais conforme as preferências do aluno. Com os itens já concluídos outros aspectos surgiram durante a validação do sistema proposto, os quais originarão trabalhos futuros para o aprimoramento do mesmo: Adaptar o sistema permitindo o usuário visualizar mais de uma dimensão preferencial; Pesquisar métodos e estratégias de aprendizagem para cada uma das dezessete dimensões estudadas; e, Integrar um módulo ao sistema que permita o professor visualizar o estilo de aprendizagem de seus alunos como também indicando materiais instrucionais preferências de acordo com o respectivo estilo.

\section{Referências}

[1] M. E. B. de Almeida. Tecnologia e educação a distância: abordagens e contribuições dos ambientes digitais e interativos de aprendizagem.2008. Disponível em: <http://www.anped.org.br/reunioes/26/trabalhos/ mariaelizabethalmeida.rtf.> Acesso em: 14 abr.2010.

[2] K. A. Butler. Estilos de Aprendizagem: as dimensões psicológica, afetiva e cognitiva. Traduzido por Renata Costa de Sá Bonotto e Jorge Alberto Reichert. Porto Alegre: Editora da U-
FRGS, 2003.

[3] L. C. B. Cavelucci. Estilos de Aprendizagem: em busca das diferenças individuais. 2006, p.10-12. Disponível em: $<$ http://www.iar.unicamp.br/disciplinas/am540_2 003/lia/estilos_de_aprendizagem.pdf $>$. Acesso em: 14 abr 2010.

[4] R. M. Felder. Reaching the Second Tier: learning and teaching styles in college science education. Journal of College Science Teaching, v.23, n.5, p. 286-290, 1993. Disponível em:< http://www.ncsu.edu/felder-

public/Papers/Secondtier.html). Acesso em: 29 abr. 2010.

[5] P. Honey, A. Mumford. The Learning Styles helper's guide. Maldenhead Berks: Peter Honey Publications, 2000.

[6] D. A. Kolb. Experiential Learning: experience as the source of learning and development. Englewood Cliffs: Prentice-Hall, 1984.

[7] B. K. Given. The overlap between brain research and research on learning style, In S. J. Armstrong et al. (Eds.), Learning Styles: Realibility \& Validity, Proceedings of the $7^{\text {th }}$ Annual ELSIN Conference. Ghent: Ghent University. Belgium \& ELSIN, 2002, p. 173-178.

[8] V. Lindemann. Estilos de Aprendizagem: buscando a sinergia. (2008, p.165). Tese de Doutorado em Informática na Educação. Porto Alegre: UFRGS.

[9] P. M. Mozzaquatro. Adaptação do Mobile Learning Engine. Moodle (MLE MOODLE) aos Diferentes Estilos Cognitivos utilizando Hipermídia Adaptativa. (2010, p. 150). Dissertação de Mestrado em Ciência da Informática. Santa Maria: UFSM. 